\title{
Dental Implant-supported Rehabilitation on a Vascular-grafted Mandible: A Long-term and Multidisciplinary Approach
}

\author{
Rafael S de Molon ${ }^{1}$, Mário FR Gabrielli ${ }^{2}$, Rogério Margonar ${ }^{3}$, Jônatas C Esteves ${ }^{4}$, Thallita P Queiroz ${ }^{5}$, Marisa AC Gabrielli ${ }^{6}$, \\ Eloisa M Boeck ${ }^{7}$, Cláudio Marcantonio ${ }^{8}$, Elcio Marcantonio ${ }^{9}$
}

\begin{abstract}
Background: Facial trauma may lead to severe fractures and loss of bone and dental structures. Depending on the magnitude of the trauma, whole segments of the jaws may be lost due to comminution, exposure of internal structures, or infection. The treatment of such lesions can be challenging, and success is often hindered by unsatisfactory esthetic, phonetic, and functional outcomes. The aim of this case report was to describe a multidisciplinary approach for implant rehabilitation in a complex case of trauma in the maxillofacial region in a young patient. Case description: A 22-year-old female patient was referred with extensive loss of soft and hard tissues in the mandible as well as loss of several teeth due to facial trauma. The patient was submitted to innumerous surgical reconstructive procedures throughout years of treatment, which included vascularized soft and hard tissue grafts, installation of dental implants, Le Fort I osteotomy for maxillary repositioning, and subsequent implant-supported prosthetic rehabilitation.

Conclusion: A 24-year follow-up of comprehensive surgical and prosthodontic reconstruction of a facial injury with several fractures due to motorcycle accidents using the multidisciplinary approach was described. The multidisciplinary approach employed in this case demonstrated successful outcomes for the reconstruction of the maxillofacial region after extensive facial trauma. After six years postoperatively no complications with the rehabilitation treatment has been detected to the present date.

Clinical significance: The multidisciplinary treatment planning employed was able to restore masticatory function and facial esthetics, enhancing patient quality of life and satisfaction.

Keywords: Dental implant, Le Fort osteotomy, Mandibular fractures, Maxillofacial surgery, Vascular grafting.

World Journal of Dentistry (2019): 10.5005/jp-journals-10015-1656
\end{abstract}

\section{INTRODUCTION}

Facial trauma may lead to severe fractures and loss of bone and dental structures., ${ }^{1,2}$ The leading causes of facial trauma and/ or fractures are collision of motorcycle and vehicle. ${ }^{3}$ Other less frequent causes are related to gunshot, sports, and fall. ${ }^{3}$ Depending on the magnitude of the trauma, whole segments of the jaws may be lost due to comminution, exposure of internal structures, or infection. ${ }^{4,5}$ The treatment of such lesions can be challenging, and success is often hindered by unsatisfactory esthetic, phonetic, and functional results, which can directly impair the patient's social skills and quality of life. ${ }^{6-9}$ In cases of severe trauma, the patient should be assessed with a multidisciplinary team approach to avoid any significant complication, decrease functional disability, and reduce cosmetic deformity that this kind of injury might result in the patient. ${ }^{1}$

The aim of this clinical report was to describe a multidisciplinary team approach of a patient with extensive loss of soft and hard tissues in the mandible as well as loss of several teeth due to facial trauma. The patient was submitted to several surgical reconstructive procedures throughout years of treatment, which included vascularized soft and hard tissue grafts, installation of dental implants, and subsequent implant-supported prosthetic rehabilitation.

\section{Case Description}

In February 1995, a 22-year-old female patient was involved in a motorcycle accident and had her face directly against the back of another vehicle. Injuries included cranial trauma, Le Fort I and
$1,2,6$ Department of Diagnosis and Surgery, School of Dentistry at Araraquara, Sao Paulo State University-UNESP, Araraquara, São Paulo, Brazil

${ }^{3,5,7-9}$ Department of Health Sciences, Centro Universitario de Araraquara, UNIARA Dental School, Araraquara, São Paulo, Brazil

${ }^{4}$ Department of Clinical Dentistry, Federal University of Rio de Janeiro-UFRJ, Rio de Janeiro, Brazil

Corresponding Author: Rafael S de Molon, Department of Diagnosis and Surgery, School of Dentistry at Araraquara, Sao Paulo State University-UNESP, Araraquara, São Paulo, Brazil, Phone: +55 16 33016300, e-mail: rafael.molon@unesp.br

How to cite this article: de Molon RS, Gabrielli MFR, Margonar R, et al. Dental Implant-supported Rehabilitation on a Vascular-grafted Mandible: A Long-term and Multidisciplinary Approach. World J Dent 2019;10(4):310-315.

Source of support: Nil

Conflict of interest: None

sagittal maxillary fractures; fracture of the left zygomaticomaxillary complex; extrusion of the posterior dentoalveolar segment; loss of teeth \#7,8,9,10, and 11, loss of the mandibular segment correspondent to the symphysis and body on both sides, and injury of the related mucosa and skin (Fig. 1).

During the first 20 days after the accident, the patient remained in the intensive care unit (ICU) for treatment and monitoring of her neurological status. According to the patient's report, after being released from the ICU, she was submitted to the surgical reduction of the sagittal maxilla and the left zygomaticomaxillary complex 

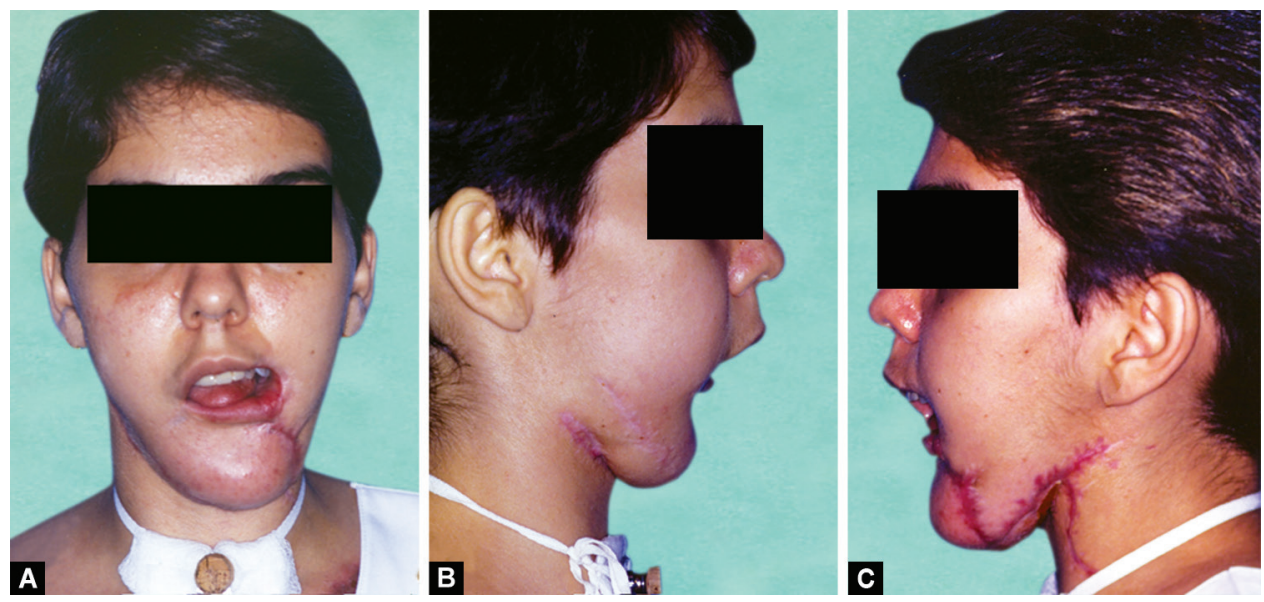

Figs. 1 A to C: Initial facial aspect after intensive care
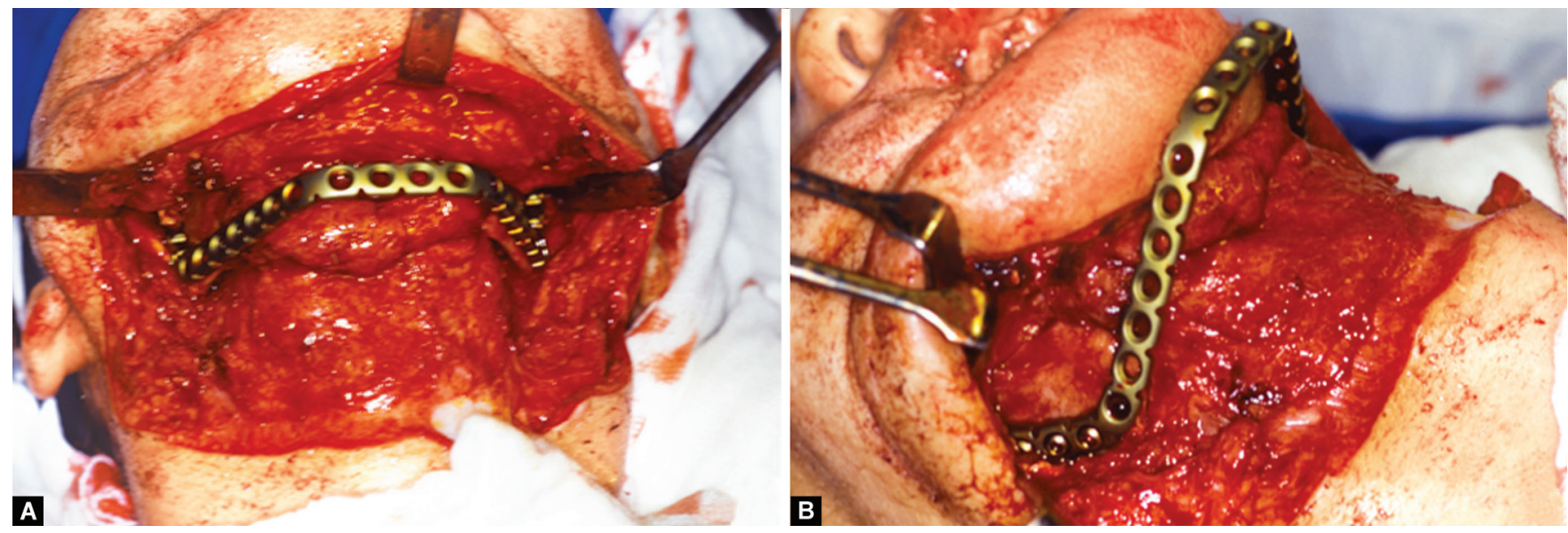

Figs $2 \mathrm{~A}$ and B: (A) Frontal; (B) Lateral view of reconstruction titanium plate fixed on the remaining mandibular bone
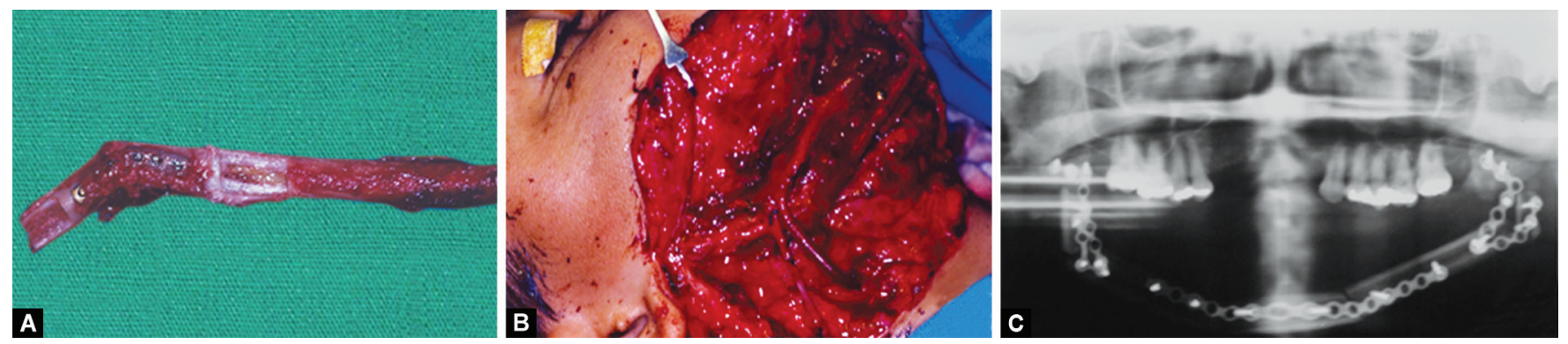

Figs. 3A to C: Tibial microvascular flap for mandibular reconstruction

fractures, as well as the removal of the comminuted mandible fragments. Reconstructive facial surgeries were not carried out at that stage as priority was given to the treatment of other urgent injuries throughout the body. On a subsequent occasion, the patient searched for treatment at the Department of Buco-maxillofacial Surgery at the Araraquara Dental School-UNESP, where she was admitted with severe sequels from the previous treatment.

Since vascular grafting was discarded at this time, the initial reconstructive approach consisted of repositioning and stabilization of the remaining bone segments with a $2.4 \mathrm{~mm}$ titanium plate for mandibular reconstruction, which was rigidly fixed bilaterally on the remaining mandibular bone next to the ascending rami (Fig. 2). This procedure was intended to properly reposition the soft tissue and to create space for a future bone grafting receptor site. In order to reconstruct the mental region and the floor of the mouth, a free skin graft was performed.

The next procedure, carried out in January 1997, was the first attempt for the mandible reconstruction using a vascularized bone graft harvested from the right-tibia as a donor site. The fibular artery microvascular flap was bilaterally fixed to the mandibular bone segments and anastomosed to the facial veins and arteries. Postoperative vascular thrombosis led to early loss of the flap and subsequent failure of the graft. A second reconstruction tentative was carried out using a scapula bone graft with a pectoral muscle flap, which also failed due to a lack of graft integration to the residual bone. Lastly, the third surgical reconstructive attempt was a left-tibia pediculate microvascular flap, which was anastomosed to the right ascending pharyngeal artery (Fig. 3). This vascular 
graft integrated well to the remaining bone at the receptor site, which allowed for reconstruction of the mandibular contour with vital bone. After 18 months, seven $4 \times 10 \mathrm{~mm}$ dental implants (Conexao Sistemas de Proteses, Aruja, SP, Brazil) were inserted in the reconstructed mandibular bone and remained inactive for osseointegration during 12 months (Figs $4 \mathrm{~A}$ and $\mathrm{B}$ ).

After the placement of dental implants in the mandible, another surgical procedure was carried out in the maxilla in order to correct the left posterior dentoalveolar segment extrusion. The surgery was planned with the aid of a panoramic radiograph and articulated cast models of the jaws. After a $6 \mathrm{~mm}$ intrusion of the segmented superior cast from tooth \#6-10, an acrylic stent was built to guide the positioning of the bone segment during the surgery. This procedure was carried out under local anesthesia. After anesthetic infiltration and the raising of the flap, a horizontal ostectomy running $5 \mathrm{~mm}$ superiorly to the apices of teeth \#6, 7, 8,9 , and 10 was performed and followed at both edges by two vertical ostectomies towards the alveolar crest (Fig. 4C). Through the buccal opening created by the planned $6 \mathrm{~mm}$ ostectomy on the fixed segment, the palatal bone was also sectioned with the aid of oscillating saws and conical burs. After mobilization, the sectioned bone segment was adapted to the acrylic stent and superiorly positioned in alignment with the opposing side to be rigidly fixed with titanium plates and screws (Fig. 6D). The surgical stent and the titanium fixtures were removed 10 and 90 days, respectively, after surgery.

At the time of reopening for placement of the healing caps, one implant was not osseointegrated. After removal of the failing implant, impression procedures were carried out for the installation of the patient's full-arch implant-supported restoration, which required the manufacturing of special $9 \mathrm{~mm}$ long prosthetic components (Conexao Sistemas de Proteses, Aruja, SP, Brazil). At this stage, the maxilla received a temporary removable partial prosthesis.

In 2007, although the maxilla was aligned with the sagittal plane, it revealed poor relation between the upper lip and the superior incisor teeth. Under hospitalization and general anesthesia, a Le Fort I osteotomy was carried out for superior intrusion of the maxilla in order to achieve a proper relation between the superior incisor teeth and the resting upper lip.

Having established an adequate relation between the upper and the lower jaws, in November 2009, three screw-fixed homologous cortico-medullary bone blocks were grafted onto the anterior maxilla in order to recover maxillary width for the future placement of dental implants.

Nine months after grafting, the maxillary bone was exposed again for removal of the screw fixtures and insertion of three $3.75 \times 13 \mathrm{~mm}$ (Conexao Sistemas de Protese-Aruja, São Paulo, Brazil) dental implants at the sites corresponding to teeth \#6, 8, and 10. Approximately 6 months later, an acrylic temporary bridge was installed over the maxillary implants in order to restore the patient's projection of the upper lip and anterior disocclusion guide. In August 2011, the acrylic teeth were replaced by a ceramic prosthesis, and the inferior Bränemark protocol prosthesis was redone in order to reestablish satisfactory functional and esthetic parameters (Figs 5 and 6).

Currently, the patient is under a maintenance program carried out by our rehabilitation team. Both the implants and the prosthesis
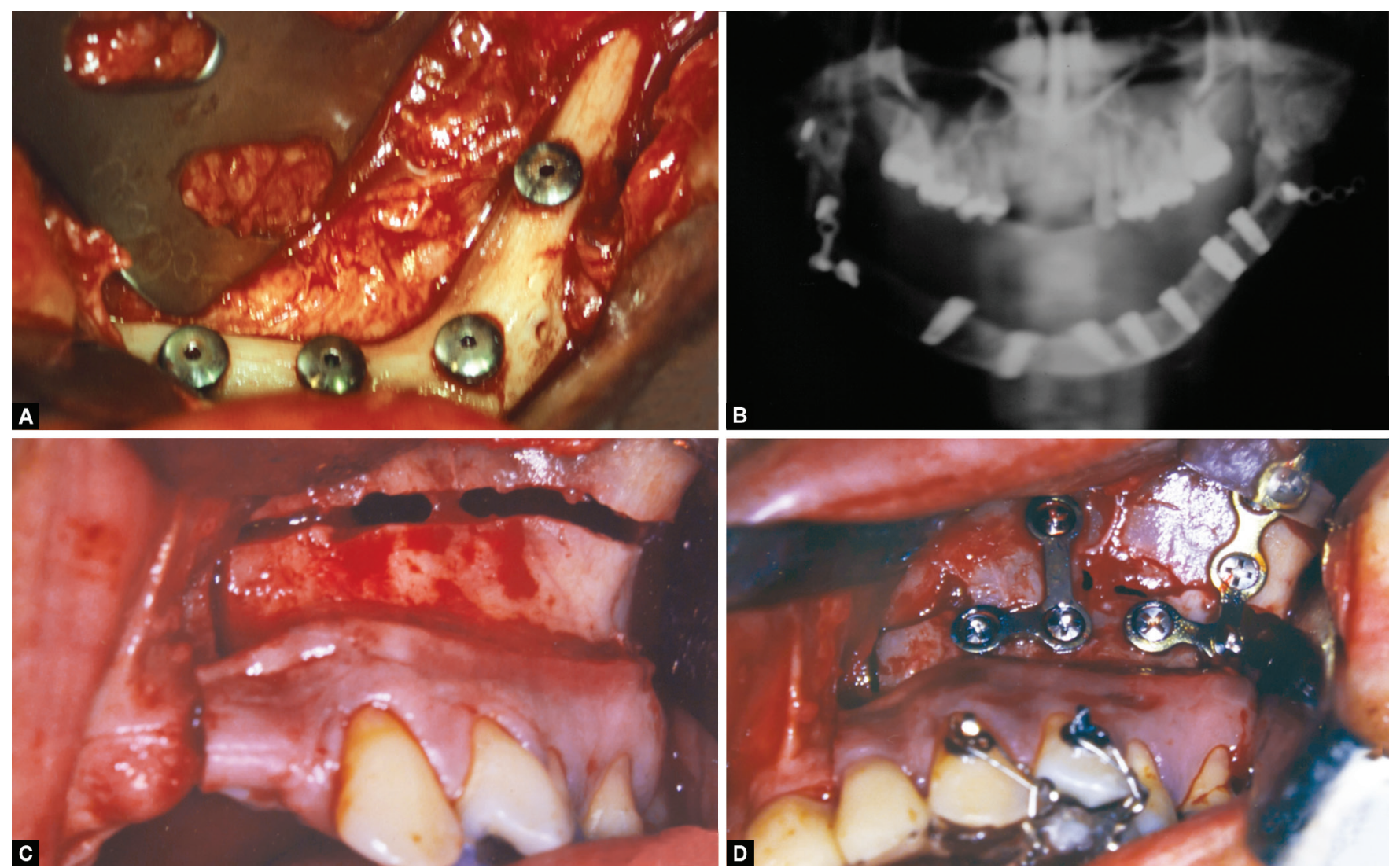

Figs 4A to D: (A and B) Dental implants installation on the tibial microvascular flap; (C and D) Intrusion of dentoalveolar segment; (A) Vertical and horizontal osteotomies; (B) Fixation of the segment with titanium plates and screws 

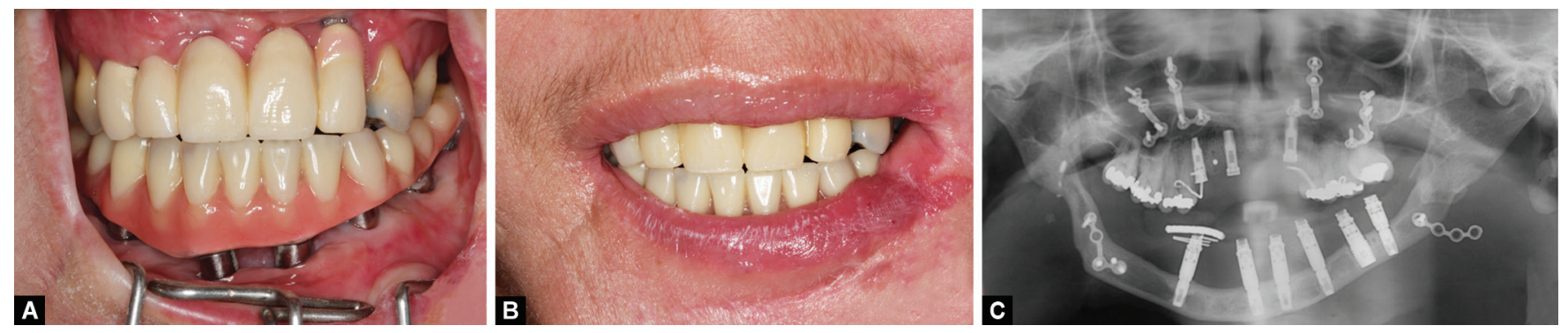

Figs 5 A to C: Final oral rehabilitation with dental implants. Full arch prosthesis in the mandible and a porcelain bridge on the maxilla
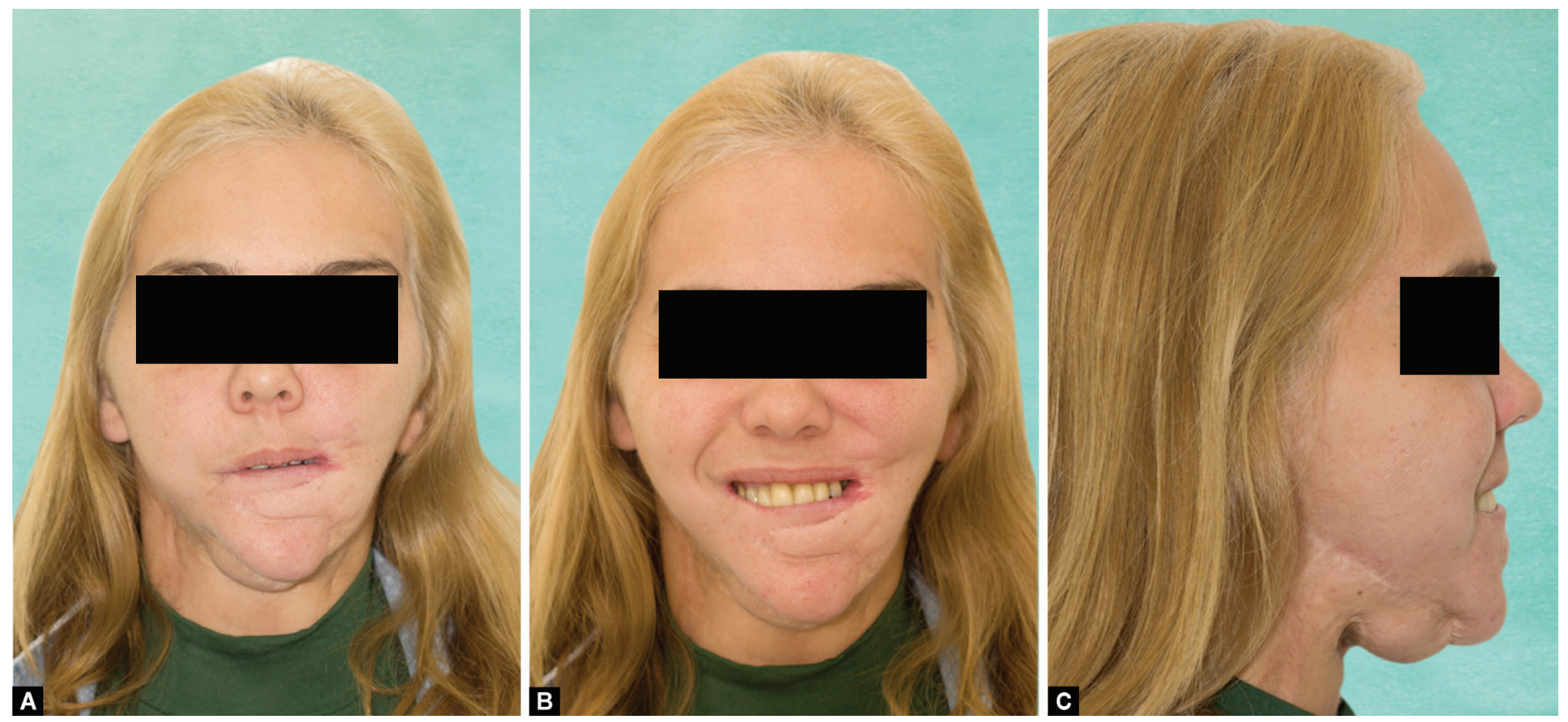

Figs $6 \mathrm{~A}$ to C: Final facial aspect after oral rehabilitation

are in good condition, and no complication with the rehabilitation treatment has been detected to the present date. The patient still needs cosmetic reconstructive surgery to achieve satisfactory facial esthetics (Fig. 6).

\section{Discussion}

This report presents a clinical case in which a long-term and multidisciplinary treatment was carried out to reestablish phonetic and masticatory skills as well as esthetics to a patient severely injured in the mandible due to motorcycle accidents. Consequences from the trauma were not limited to fractures but also included the loss of bone, muscular, and cutaneous tissues in the body of the mandible, which required the combination of specific reconstruction techniques. Early treatment of the facial traumas was not possible due to other occurring urgencies such as upper limb fractures and neurological status monitoring. Sequels from this late approach were challenges we had to face at the initial stage of the patient treatment.

Our first surgical procedure was the reduction and fixation of the maxilla and the zygomaticomaxillary complex fractures. Simultaneously, a titanium reconstruction plate was used to bridge and stabilize the remaining mandibular segments and prevent soft tissue from collapsing during the reconstruction planning period. Facial bone defects up to $5 \mathrm{~mm}$ at its larger portion have been reported to be treated with free autologous grafts. ${ }^{10,11}$ However, long-term success of these grafts are sensitive to favorable conditions, such as sufficient remaining bone structure for fixation, adequate blood supply, and the presence of soft tissue for primary coverage of the wound, all of which may be compromised at the receptor site in cases of extensive bone defects. ${ }^{12,13}$ The use of conventional free grafting was discarded for this case due to the extensive loss of soft and hard tissues, which suggested that a microvascular grafting procedure would be more adequate.

Vascular bone grafts (VBG) have a high success rate for large reconstructions of the facial bones ${ }^{14-16}$ due to its highly regenerative potential, as the immediate anastomosing of the graft vessels to the facial arteries allows for prompt reestablishment of the vascular supply. ${ }^{10,13}$ Usually harvested from the iliac crest, fibula, or the scalp, the VBG can be surrounded by soft tissue and adapted to the receptor site before the fixture. ${ }^{17}$ Despite its advantages, harvesting, fixation, and anastomosing in VBG are complex procedures, which are subject to complications and require the expertise of an experienced team. Failure in soft tissue perfusion due to thrombosis or deficient anastomosing may lead to necrosis of the whole graft and proneness to infection at the receptor site. ${ }^{18}$ In the present case, such conditions led to the failure of the graft in the first two reconstruction attempts. Success was achieved only at the third attempt when a fibula flap anastomosed to the ascending pharyngeal artery was used. 
In individuals with advanced oral and maxillofacial trauma, the loss of the mandibular and maxillary bones and teeth results in both esthetic and functional abnormalities. Immediate and comprehensive management involving multiple disciplines is mandatory to effectively restore the injured hard and soft tissues, reestablish the function and esthetics, and allow the patient to regain their self-esteem. ${ }^{19}$ The current literature shows that rehabilitation of a patient with facial trauma is a long-term and challenging procedure. ${ }^{20}$ VBG play an important role in long-term success due to their blood supply, adequate structure and shape, and biocompatibility. This bone has the capacity to integrate dental implants with high implant success and survival rates. ${ }^{21,22}$

Although facial anatomy was restored with vascular bone grafting, a further goal of the treatment proposed by us was the rehabilitation of the lost teeth. Several authors have reported the viability of dental implants in vascularized graft areas, where, due to regenerative properties identical to the native bone, they show success rates similar to implants placed in nonvascular bone grafts. ${ }^{23-26}$ Dental implant placement in these areas allows for rehabilitation with implant-supported fixed bridges, which should restore masticatory and phonetic functions to the patient. In this case, the implants were found to be osseointegrated, which might indicate that VBG is a reliable alternative to reconstruct the mandible after facial trauma.

The installation of 7 implants in the reconstructed mandible is allowed for complete rehabilitation of the inferior jaw with Bränemark protocol prosthesis. However, the trauma-related poor positioning of the maxilla prevented the reestablishment of an adequate relation between the upper and the lower jaws. Both orthognathic surgeries that followed are allowed for sufficient interocclusal space, proper horizontal alignment, and adequate labial support for a functionally acceptable dental occlusion. Lastly, the grafting of homologous bone blocks allowed for the rehabilitation of the anterior maxilla with dental implant-supported fixed bridges. The use of a homologous graft was meant to spare the patient from any other extra surgical procedures, such as the harvesting of bone needed for autologous grafting.

Currently, there is not a specific treatment modality that could be addressed for mandibular reconstruction after major facial trauma, and evaluation of the patient's situation plays a pivotal role in the treatment plan. Nevertheless, the implant-supported prosthesis can definitively improve the chewing capacity of the patient and therefore enhance the related quality of life. ${ }^{20}$ Periodic follow-ups are mandatory for observing the long-term results of this kind of reconstruction.

This case report was initiated 24 years ago, and at that time we have chosen such approaches because it was the most recommended approach to treat severe facial trauma. However, nowadays there have been increased scientific advancements in the field of oral and maxillofacial surgery, and we can speculate that with the aid of computer-aided design and computer-aided manufacturing prefabricated titanium mesh combined, with autogenous iliac cancellous bone graft would result in a more predictable outcome to reconstruct the mandibular bone. Primary would closure with such techniques would favor the final outcome of the case. Furthermore, advances with new developments in bone morphogenetic protein and stem cells would probably favor the healing of bone tissue and improve clinical outcomes. Moreover, improvements and advancement in the development of new implant surfaces (with new surfaces able at accelerating the osseointegration process), new prosthesis design, and materials could also benefit the patient for a more predictable, reliable, and faster treatment.

\section{Conclusion}

A 24-year follow-up of comprehensive surgical and prosthodontic reconstruction of a facial injury with several fractures due to motorcycle accidents using a multidisciplinary approach was described. The principles involved to manage this case were vascularized bone graft for mandible reconstruction, implant placement, Le Fort I osteotomy, and finally, prosthetic rehabilitation. The importance of a multidisciplinary approach in treatment planning and execution in such situations is emphasized to achieve successful clinical outcomes.

\section{Clinical Significance}

The multidisciplinary treatment planning employed was able to restore masticatory function and facial esthetics, enhancing patient quality of life and satisfaction.

\section{Informed Consent}

Informed consent was obtained from the participant included in the study.

\section{References}

1. Ricketts S, Gill HS, Fialkov JA, et al. Facial Fractures. Plast Reconstr Surg 2016;137:424e-444e. DOI: 10.1097/01.prs.0000475760. 09451.49.

2. Schneider D, Kammerer PW, Schon G, et al. Etiology and injury patterns of maxillofacial fractures from the years 2010 to 2013 in Mecklenburg-Western Pomerania, Germany: A retrospective study of 409 patients. J Craniomaxillofac Surg 2015;43:1948-1951. DOI: 10.1016/j.jcms.2015.06.028.

3. Erdmann $\mathrm{D}$, Follmar $\mathrm{KE}$, Debruijn $\mathrm{M}$, et al. A retrospective analysis of facial fracture etiologies. Ann Plast Surg 2008;60:398-403. DOI: 10.1097/SAP.0b013e318133a87b.

4. Guven Y, Zorlu S, Cankaya AB, et al. A Complex Facial Trauma Case with Multiple Mandibular Fractures and Dentoalveolar Injuries. Case Rep Dent 2015;2015:301013. DOI: 10.1155/2015/301013.

5. Park KP, Lim SU, Kim JH, et al. Fracture patterns in the maxillofacial region: a four-year retrospective study. J Korean Assoc Oral Maxillofac Surg 2015;41:306-316. DOI: 10.5125/jkaoms.2015.41.6.306.

6. Arcuri F, Brucoli M, Baragiotta N, et al. The role of fat grafting in the treatment of posttraumatic maxillofacial deformities. Craniomaxillofac Trauma Reconstr 2013;6:121-126. DOI: 10.1055/s0033-1333877.

7. Chung KJ, Kim YH, Kim TG, et al. Treatment of complex facial fractures: clinical experience of different timing and order. J Craniofac Surg 2013;24:216-220. DOI: 10.1097/SCS.0b013e318267b6f7.

8. Khader R, Wallender A, Van Sickels JE, et al. Secondary reconstruction of panfacial fractures. Oral Maxillofac Surg 2014;18:99-109. DOI: 10.1007/s10006-013-0403-8.

9. de Avila ED, de Molon RS, Loffredo LC, et al. Health-related quality of life and depression in patients with dentofacial deformity. Oral Maxillofac Surg 2013;17:187-191. DOI: 10.1007/s10006-012-0338-5.

10. Chiapasco M, Zaniboni M, Boisco M. Augmentation procedures for the rehabilitation of deficient edentulous ridges with oral implants. Clin Oral Implants Res 2006;17(Suppl 2):136-159. DOI: 10.1111/j.16000501.2006.01357.x.

11. Foster RD, Anthony JP, Sharma A, et al. Vascularized bone flaps vs nonvascularized bone grafts for mandibular reconstruction: an outcome analysis of primary bony union and endosseous 
implant success. Head Neck 1999;21:66-71. DOI: 10.1002/(SICI)10970347(199901)21:1<66::AID-HED9>3.0.CO;2-Z.

12. Schmelzeisen $\mathrm{R}$, Schliephake $\mathrm{H}$. Interdisciplinary microvascular reconstruction of maxillary, midfacial and skull base defects. J Craniomaxillofac Surg 1998;26:1-10. DOI: 10.1016/S10105182(98)80028-0.

13. Kumar BP, Venkatesh V, Kumar KA, et al. Mandibular Reconstruction: Overview. J Maxillofac Oral Surg 2016;15:425-441. DOI: 10.1007/ s12663-015-0766-5.

14. Hidalgo DA, Rekow A. A review of 60 consecutive fibula free flap mandible reconstructions. Plast Reconstr Surg 1995;96:585-596, discussion 597-602, 10.1097/00006534-199509000-00010.

15. Jones NF, Monstrey S, Gambier BA. Reliability of the fibular osteocutaneous flap for mandibular reconstruction: anatomical and surgical confirmation. Plast Reconstr Surg 1996;97:707-716, discussion 717-708, 10.1097/00006534-199604000-00003.

16. Taylor GI, Corlett RJ, Ashton MW. The Evolution of Free Vascularized Bone Transfer: A 40-Year Experience. Plast Reconstr Surg 2016;137:1292-1305. DOI: 10.1097/PRS.0000000000002040.

17. Chen YB, Chen HC, Hahn LH. Major mandibular reconstruction with vascularized bone grafts: indications and selection of donor tissue. Microsurgery 1994;15:227-237. DOI: 10.1002/micr. 1920150403.

18. Smolka W, lizuka T. Surgical reconstruction of maxilla and midface: clinical outcome and factors relating to postoperative complications. J Craniomaxillofac Surg 2005;33:1-7. DOI: 10.1016/j.jcms.2004. 09.001.

19. Yamano S, Nissenbaum M, Dodson TB, et al. Multidisciplinary treatment for a young patient with severe maxillofacial trauma from a snowmobile accident: a case report. J Oral Implantol 2010;36:141-144. DOI: 10.1563/AAID-JOI-D-09-00058R1.

20. Kokat AM, Ercocen AR, Karayazgan-Saracoglu B. Simultaneous Implant Placement in a Mandibular Defect Reconstructed With a Free Fibula Graft and Fabrication of a Fixed Metal Porcelain Prosthesis. J Craniofac Surg 2018;29:1307-1311. DOI: 10.1097/SCS.0000000000004526.

21. Carbiner R, Jerjes W, Shakib K, et al. Analysis of the compatibility of dental implant systems in fibula free flap reconstruction. Head Neck Oncol 2012;4:37. DOI: 10.1186/1758-3284-4-37.

22. Schepers RH, Kraeima J, Vissink A, et al. Accuracy of secondary maxillofacial reconstruction with prefabricated fibula grafts using 3D planning and guided reconstruction. J Craniomaxillofac Surg 2016;44:392-399. DOI: 10.1016/j.jcms.2015.12.008.

23. Beumer 3rd J, Roumanas E, Nishimura R. Advances in osseointegrated implants for dental and facial rehabilitation following major head and neck surgery. Semin Surg Oncol 1995;11:200-207. DOI: 10.1002/ ssu. 2980110305.

24. Chiapasco M, Colletti G, Romeo E, et al. Long-term results of mandibular reconstruction with autogenous bone grafts and oral implants after tumor resection. Clin Oral Implants Res 2008;19: 1074-1080. DOI: 10.1111/j.1600-0501.2008.01542.x.

25. Viscioni A, Rigo L, Franco M, et al. Reconstruction of severely atrophic jaws using homografts and simultaneous implant placement: a retrospective study. J Oral Implantol 2010;36:131-139. DOI: 10.1563/ AAID-JOI-D-09-00025.

26. Jackson RS, Price DL, Arce K, et al. Evaluation of Clinical Outcomes of Osseointegrated Dental Implantation of Fibula Free Flaps for Mandibular Reconstruction. JAMA Facial Plast Surg 2016;18:201-206. DOI: 10.1001/jamafacial.2015.2271. 